\title{
periferio
}

\section{IMPLICAÇÕES DOS DISTÚRBIOS RESPIRATÓRIOS DO SONO EM ALUNOS COM DEFICIÊNCIA INTELECTUAL: REVISÃO SISTEMÁTICA}

\author{
Miriam Adalgisa Bedim Godoy ${ }^{1}$ \\ Universidade Estadual do Centro-Oeste \\ Maria Amelia Almeida ${ }^{2}$ \\ Universidade Federal de São Carlos
}

\section{Resumo}

Nos últimos anos, as pesquisas sobre os distúrbios respiratórios do sono em crianças e adolescentes com desenvolvimento típico têm ampliado. As pesquisas constataram que o sono de má qualidade interfere nas competências físicas e intelectivas do ser humano. Como seria a influência desse distúrbio em educandos com deficiência intelectual? 0 estudo teve por objetivo verificar e analisar nas publicações científicas a influência dos distúrbios respiratórios do sono em escolares com deficiência intelectual. Trata-se um delineamento sistemático de cunho qualitativo. O levantamento foi realizado no período que compreende os anos de 2000 a 2016. Os artigos estavam indexados bases de dados EBSCOhost, DOAJ, ERIC e SciELO Brasil. Os descritores utilizados foram i) distúrbios respiratórios do sono e dificuldade de aprendizagem; ii) distúrbios respiratórios do sono e deficiência intelectual; iii) distúrbios respiratórios do sono e etiologia da deficiência intelectual; iv) distúrbios respiratórios do sono e déficit intelectual. A busca também foi realizada em língua inglesa, a saber: i) sleep-disordered breathing and learning disability; ii) sleep-disordered breathing and Intellectual disability; iii) sleepdisordered breathing and Etiology intellectual disability; iv) sleep-disordered breathing and Intellectual deficit. Com base nesses descritores, foram encontradas 43 pesquisas. Entretanto, considerando-se os requisitos elegidos para este estudo, apenas sete artigos compuseram o corpus final de análise. Desses, quatro eram estudos experimentais, dois de revisões de literatura e um do tipo longitudinal. As pesquisas revelaram que a influência dos distúrbios do sono junto às crianças e aos adolescentes com deficiência intelectual é recente e limitada, porém, tais distúrbios respiratórios do sono interferem significativamente na qualidade de vida, bem como nos aspectos de desenvolvimento e aprendizagem dos alunos com deficiência intelectual. Esse levantamento ressaltou a necessidade de pesquisas brasileiras, sobretudo em Educação Especial, de se preocuparem em inserir os distúrbios respiratórios do sono nos estudos junto a essa população escolar.

Palavras-chave: educação especial; deficiência intelectual; distúrbios respiratórios do sono; revisão sistemática

\footnotetext{
1 Doutoranda em Educação Especial - UFSCar. Professora do Departamento de Pedagogia Irati. Experiência em educação Especial e Inclusiva. Universidade Estadual do Centro-Oeste UNICENTRO. miriamadalgisa@terra.com.br

2 PhD em Educação Especial. Docente do Programa de Pós-Graduação em Educação Especial da Universidade Federal de São Carlos - SP. ameliama@terra.com.br
} 


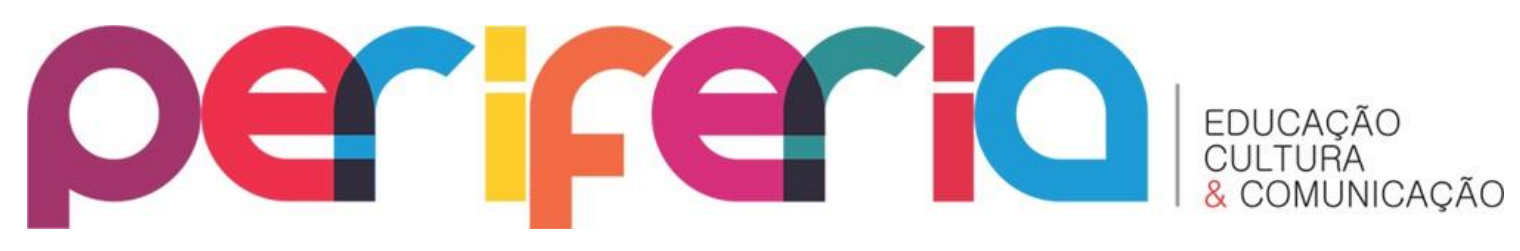

\title{
IMPLICATIONS OF SLEEP-DISORDERED BREATHING IN STUDENTS WITH INTELLECTUAL DISABILITIES: SYSTEMATIC REVIEW
}

\begin{abstract}
In recent years research on sleep-disordered breathing in children and adolescents with typical development have been expanded. Research has shown that poor quality sleep interferes with the physical and intellectual abilities of the human being. What then would be the influence of this disorder on students with intellectual disabilities? The study aims to verify and analyze the influence of respiratory sleep disorders in students with intellectual disabilities in scientific publications. This is a systematic design of a qualitative nature. The survey was conducted from 2000 to 2016 . The articles were indexed in the main databases: EBSCOhost, DOAJ, ERIC and SciELO Brazil. The en descriptors were: Sleep-disordered breathing and learning disability; Sleep-disordered breathing and Intellectual disability; Sleep-disordered breathing and Etiology intellectual disability; Sleepdisordered breathing and Intellectual deficit. The search was also conducted in English. Based on these descriptors, forty-three surveys were located. However, only seven articles met the requirements chosen for this study. From these articles, four are experimental type of studies, two of them were literature review and one was longitudinal type of study. The review revealed that research on the influence of sleep disorders on children and adolescents with intellectual disabilities is recent and still very limited and the sleepdisordered breathing significantly interferes with life quality as well as developmental and learning aspects of students with intellectual disabilities. This study underscores the need for Brazilian research in respiratory sleep disorders in the area of special education.
\end{abstract}

Keywords: special education; intellectual disability; sleep-disordered breathing; systematic review 


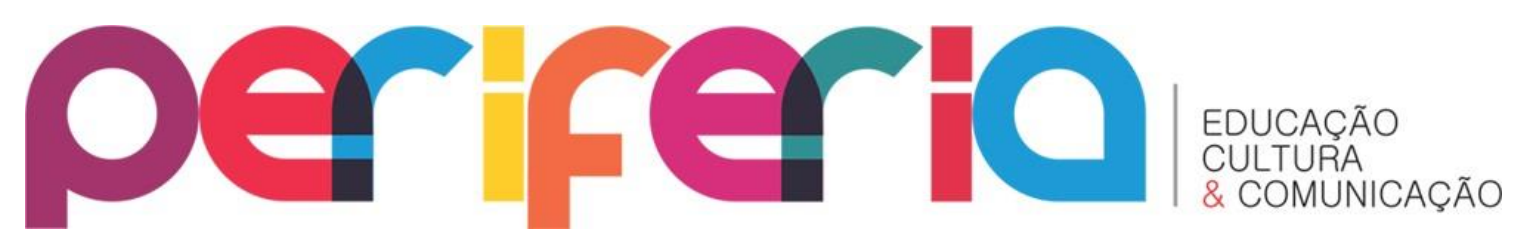

\section{INTRODUÇÃO}

Nos últimos anos têm ocorrido um aumento expressivo de reconhecimento dos distúrbios do sono em crianças com desenvolvimento típico (DI FRANCESCO, 2004). Apesar disso, ainda, há uma escassez de dados sobre tal problema, bem como como há uma falta de treinamento de profissionais médicos para identificar a causa e encaminhar para procedimentos adequados (LUMENG; CHERVIN, 2008).

Estudos contemporâneos verificaram que crianças pequenas com interrupções frequentes do sono podem ter dificuldade de despertar pela manhã, resultando em associações comportamentais (OWENS, 2010). 0 exame mais indicado para verificar a arquitetura do sono é a polissonografia possibilita avaliar o tipo, frequência e duração das apneias; características do ronco; grau de dessaturação do oxigênio; avaliação das arritmias cardíacas; fragmentação do sono e severidade do quadro (MACEDO; COLOMBINI; PINTO, 2010).

Para Martinez (1999) o sono "sonegado" ou a falta de sono, com o tempo, gera um "débito de sono", comprometendo as competências físicas e intelectivas. 0 autor supracitado esclarece que tal privação interfere nas habilidades do dia a dia, como a memória, a atenção, o raciocínio lógico, a resolução de operações aritméticas, planejamento, linguagem e aprendizado escolar.

Uma parcela significativa de crianças e adolescentes com desenvolvimento típico, inseridos no contexto escolar, apresenta problemas de sono. Martinez (1999) faz referência ao relatório americano, o qual ressalta que os problemas do sono são identificados em 20 a 30 por cento de crianças entre um a cinco anos. Para este autor, em nível de aprendizado escolar, essa população está inserida na etapa da Educação Infantil. Porém, se esses problemas não forem tratados permanecerão ao longo do Ensino Fundamental e Médio - etapas essas que requerem grande esforço físico e 


\section{periferio}

intelectual para apreensão e domínio dos conceitos científicos (MARTINEZ, 1999).

Para Martinez (1999), os principais sintomas apresentados pelas crianças com problemas do sono são irritação, déficit de atenção, instabilidade de humor e dificuldade de socialização e cooperação. 0 conjunto de sinais e sintomas, de acordo com esse autor, interfere no desempenho escolar, comprometendo o processo educacional e possibilidades futuras.

Para Rosinha (2000), o sono tem sido objeto de estudo da ciência desde o final do século XIX. Outrora, pela inexistência de tecnologia que possibilitasse uma mensuração mais pontual e real do sono, utilizava-se 0 método de observação para a compreensão desse fenômeno.

Uma descrição aprofundada dos distúrbios obstrutivos do sono (síndrome da apneia e da hipopneia) foi realizada em 1892 por William Osler. Em seu estudo, o médico fez referência ao aumento do tecido linfóide com repercussões no desenvolvimento físico e mental da criança. As características do sono apresentadas por ele são observadas na atualidade, a saber: sono perturbado, respiração ruidosa e dificultosa com a presença de pausas respiratórias e profundas inspirações que, muitas vezes, levam a pessoa a despertar com a sensação de sufocamento e falta de ar (VALERA; DEMARCO; ANSELMO-LIMA, 2004).

Para Spahis (1994), em 1889, um médico inglês chamado Hill descreveu o caso de um garoto que apresentava respiração bucal, paradas respiratórias durante $\mathrm{o}$ sono e dificuldades escolares. 0 autor fez referência aos professores para prestarem atenção sobre a influência da obstrução nasal e da respiração oral sobre o aprendizado.

Passados oito décadas, precisamente em 1976, o médico Guilleminault e sua equipe constataram a síndrome da apneia e hipopneia obstrutiva do sono (SAHOS) por meio de exame polissonográfico em oito crianças, confirmando as descrições já mencionadas. Os pesquisadores destacaram, também, que a respiração pela boca tem sido associada às mudanças 


\section{periferio}

estruturais no rosto, conhecido como fácies adenoideanas (GUILLEMINAULT et al., 1976).

Após a publicação de Guilleminault et al. (1976) houve um crescimento exponencial nas pesquisas relacionadas aos distúrbios respiratórios do sono, como fisiopatologia, morbidade e tratamento de apneia obstrutiva do sono pediátrica (GOZAL; POPE, 2001).

Uma triagem criteriosa sobre a apneia obstrutiva do sono (OSA) foi realizada por Gozal (1998) com 297 crianças do Ensino Fundamental que apresentavam desempenho escolar abaixo da média da classe, cujo resultado revelou uma elevação pontual na prevalência de distúrbios do sono. Após a avaliação das crianças que foram tratadas, o pesquisador observou melhoras significativas em suas notas escolares no ano subsequente, enquanto que as crianças que tiveram OSA, mas que não foram tratadas, não conseguiram melhorar academicamente.

Desde então, inúmeros estudos têm sido realizados relatando a associação entre OSA e distúrbios neurocognitivos e comportamentais. Huang et al. (2007) verificaram que as crianças que roncam tendem a ter menor desempenho acadêmico do que as que não roncam, independente de ter tratamento com amigdalectomia e adenoidectomia. Em geral, para os autores, os escolares são acometidos por outros sintomas, como comportamento motor excessivo e desatenção que podem ser confundidos com transtorno de déficit de atenção e hiperatividade (TDAH).

Gottlieb, Chase, Vezina (2004) afirmaram que o ronco está mais presente em crianças de dois a cinco anos - período em que ocorre um aumento das amígdalas e adenóides, obstruindo significativamente as vias aéreas superiores. Essas crianças podem apresentar comportamentos, como terror noturno, atitudes confusas ao despertar, agitação e inquietação na cama - na tentativa de melhorar a respiração, raramente se queixam de sonolência diurna, mostrando-se muitas vezes irritadas, hiperativas e com insônia; durante o sono babam e algumas não conseguem controlar os esfíncteres (GOTTLIEB; CHASE; VEZIN, 2004). 


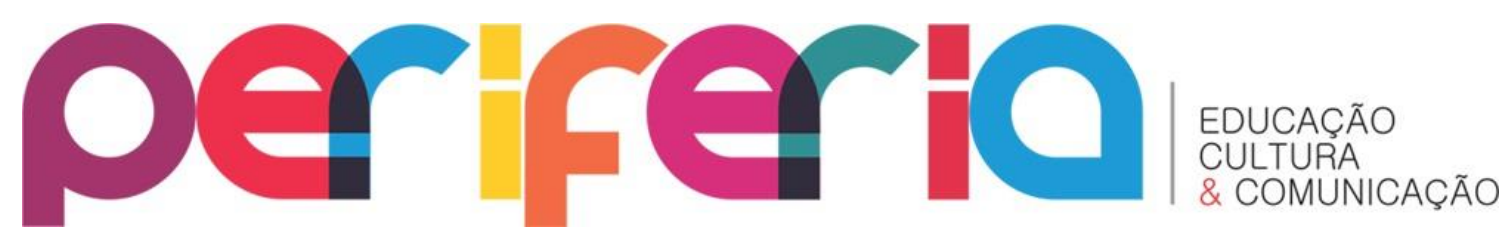

Os autores citados afirmam que crianças em idade escolar, após os seis anos, podem ainda apresentar características já mencionadas, mas com maior diversidade. Sequelas de longo prazo começam a se tornar aparentes neste grupo etário, destacando-se as dificuldades de aprendizagem, a falta de concentração e memória - principalmente a memória de curto prazo. Algumas áreas específicas de dificuldade têm sido detectadas, por exemplo, visuoespacial, resolução de problemas, memória e aritmética, assim como as funções executivas (GOTTLIEB; CHASE; VEZIN, 2004).

Na clínica pediátrica, a prevalência de distúrbios do sono e respiração oral têm se tornado muito comum. Uma pesquisa nacional realizada em 2010 verificou um pico desses distúrbios na faixa etária de quatro a sete anos (ANSELMO-LIMA; TAMASHIRO; VALERA, 2013).

0 estudo de doenças respiratórias relacionadas ao sono tem sido um continuum, o qual se verifica desde ronco até a apneia. Pesquisas atuais que indicam a prevalência de problemas respiratórios relacionados ao sono chegam a 3,3\% nas crianças, sendo que os garotos são significativamente mais frequentemente afetados (ANSELMO-LIMA; TAMASHIRO; VALERA, 2013).

$\mathrm{O}$ ronco primário consiste em um ruído normal que ocorre durante a passagem de ar pelas vias aéreas superiores, sem causar nenhum dano durante o sono. Uma situação diferente ocorre na síndrome da apneia do sono (SAOS) em crianças, ou seja, há uma obstrução parcial ou completa das vias aéreas superiores, prejudicando a ventilação normal (ANSELMO-LIMA; TAMASHIRO; VALERA 2013).

Quando a criança apresenta o diagnóstico de distúrbios respiratórios do sono, verifica-se um comprometimento do aprendizado de modo geral, dado que o sono é um importante aliado da aprendizagem. Pesquisas recentes revelam que dormir favorece a consolidação das novas informações e reorganização plástica da memória, resultando em depósito mais refinado de informações e uma busca mais efetiva (BECKER; FRANCO; ANJOS, 2013).

Diante do exposto, levantou-se a seguinte questão: Se para as crianças de desenvolvimento típico os distúrbios do sono comprometem, 


\section{periferio}

significativamente, seu desempenho biopsicossocial, como será a influência desse distúrbio em educandos Público Alvo da Educação Especial, com destaque para a deficiência intelectual?

\section{OBJETIVO}

Verificar e analisar nas publicações científicas a influência dos distúrbios respiratórios do sono (DRS) em educandos Público Alvo da Educação Especial na área intelectual.

\section{MÉTODO}

Foi realizada uma revisão sistemática da literatura (HOHENDORFF, 2009), baseada em trabalhos empíricos, nacionais e internacionais publicados no período de janeiro de 2000 a julho de 2016, referentes aos distúrbios respiratórios do sono e as suas repercussões em indivíduos com deficiência intelectual. Ademais, para a seleção dos artigos foram, também, eleitos os seguintes critérios de inclusão: estar disponível na íntegra, realizados com crianças e adolescentes, contemplar no título e resumo os descritores elegidos para esse estudo. Os critérios de exclusão foram: não contemplar o Público Alvo da Educação Especial na área intelectual, não apresentar os descritores selecionados para o levantamento, não estar disponível na íntegra e não ter sido realizado com crianças e adolescentes.

Para a realização da pesquisa eletrônica foram utilizados os seguintes descritores selecionados por meio do PubMed MeSH Thesaurus, tanto em português quanto inglês, a saber: i) Distúrbios respiratórios do sono e Dificuldade de Aprendizagem/Sleep-disordered breathing and Learning disability; ii) Distúrbios respiratórios do sono e Deficiência Intelectual/Sleepdisordered breathing and Intellectual disability; iii) Distúrbios respiratórios do sono e Etiologia da Deficiência Intelectual/Sleep-disordered breathing and Etiology intellectual disability e iv) Distúrbios respiratórios do sono e Déficit 


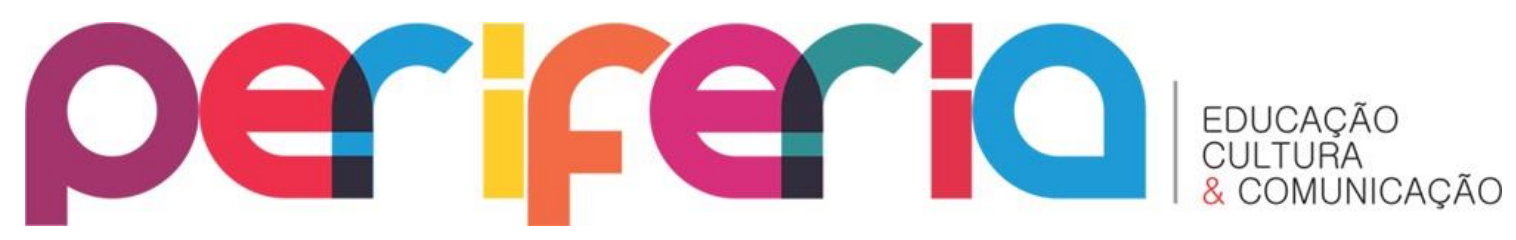

Intelectual/Sleep-disordered breathing and Intellectual deficit. Tais descritores foram eleitos dado que, de acordo com Brandau, Monteiro e Braile (2005), um descritor deve passar por um acurado controle de sinônimos, significado e importância no eixo de um assunto específico. Ratificou-se que a busca em inglês foi realizada por meio dos descritores elegidos em inglês.

As seguintes bases de dados foram consultadas: Academic Search Premier - ASP (EBSCOhost), Directory of Open Access Journals Free (DOAJ), Wiley Online Library, Educational Resources Information Center (ERIC) e Scientific Electronic Library Online (SciELO Brasil). A busca foi realizada com base em descritores representativos à temática.

Para realização da busca, recorreu-se aos descritores em português e em inglês utilizados de forma isolada e em associação.

Com base em Costa e Zoltowski (2014), os procedimentos de inclusão e exclusão dos artigos foram realizados em duas fases:

Fase 1: salutou-se importância a avaliação dos descritores, no mínimo de dois juízes independentes, os quais analisaram os resumos dos artigos elencados. Após os critérios de elegibilidade, foram avaliados os artigos selecionados;

Fase 2: primeiramente, foi realizada a extração e a análise inicial dos dados dos artigos selecionados, ou seja, os dados foram armazenados em planilhas e organizados de acordo com categorias de análise gerais (periódico, ano de publicação, autor, tipo de estudo, objetivos, resultados).

Para análise dos dados, foram produzidas categorias sobre características e conteúdos presentes nos artigos, a partir de Silveira, Enuno e Rosa (2012).

\section{RESULTADOS E DISCUSSÕES}

Com base nos critérios de inclusão e exclusão, foram localizados quarenta e três artigos $(\mathrm{N}=43)$. Contudo, a fim de cumprir com o objetivo dessa pesquisa, apenas sete estudos foram selecionados. Desses, quatro eram estudos experimentais, dois revisões de literatura e um longitudinal. Todos 


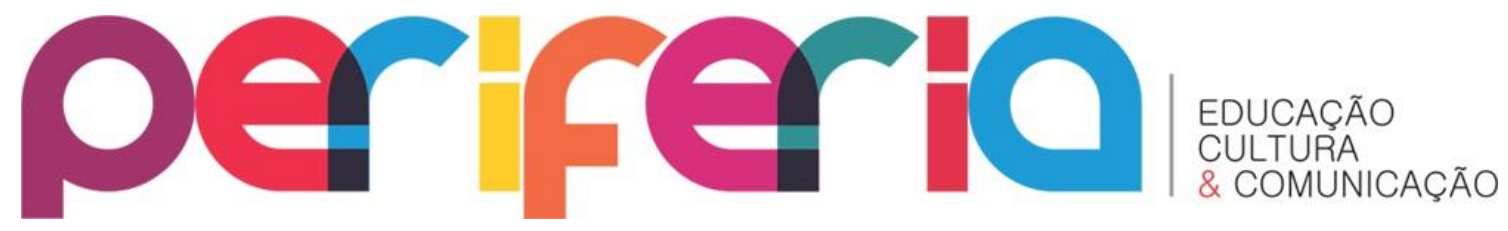

eram de veiculação internacional e estavam publicados em língua inglesa. Não foi localizado nenhum trabalho em língua portuguesa. Em relação aos campos de publicações, cinco eram da área da Educação Especial e dois da área de Medicina, conforme Quadro 1.

Quadro 1: Distribuição dos artigos selecionados de acordo com o periódico, ano de publicação, autor, tipo de estudo, objetivos e resultados. São CarlosSP, 2016

\begin{tabular}{|c|c|c|c|}
\hline $\begin{array}{c}\text { Dados do } \\
\text { Periódico/Autor }\end{array}$ & $\begin{array}{l}\text { Título/Tipo de } \\
\text { estudo }\end{array}$ & Objetivos & Resultados \\
\hline $\begin{array}{l}\text { Sleep and } \\
\text { Biological } \\
\text { Rhythms. n.13, } \\
\text { p. 323-331, } \\
2015 . \\
\text { RAHMAWATI, A } \\
\text { et al. }\end{array}$ & $\begin{array}{l}\text { Estudo } \\
\text { Longitudinal } \\
\text { Relationship } \\
\text { between sleep } \\
\text { postures and } \\
\text { sleep- } \\
\text { disordered } \\
\text { Breathing } \\
\text { parameters in } \\
\text { people with } \\
\text { Down syndrome } \\
\text { in Japan }\end{array}$ & $\begin{array}{l}\text { Determinar a relação } \\
\text { entre as posturas de } \\
\text { sono e os parâmetros } \\
\text { dos distúrbios } \\
\text { respiratórios do } \\
\text { sono, incluindo a } \\
\text { dessaturação } \\
\text { noturna de oxigênio } \\
\text { em pessoas com } \\
\text { síndrome de Down } \\
\text { no Japão. }\end{array}$ & $\begin{array}{l}\text { Foram investigados a relação } \\
\text { entre o sono existente e a } \\
\text { postura de dessaturação } \\
\text { noturna de oxigênio em pessoas } \\
\text { com síndrome de Down (SD). } \\
\text { Foram analisadas as } \\
\text { características da SD que } \\
\text { afetam essas posturas. O estudo } \\
\text { indicou que posturas de sono } \\
\text { incomum em SD pode ser } \\
\text { compensação contra distúrbios } \\
\text { respiratórios do sono (SDB), } \\
\text { especialmente, em indivíduos } \\
\text { mais jovens. }\end{array}$ \\
\hline $\begin{array}{l}\text { Behavioral } \\
\text { Sleep } \\
\text { Medicine, } \mathrm{n} . \\
12 \text {, p.1-17, } \\
2014 . \\
\text { ASHWORTH, A. } \\
\text { et al. }\end{array}$ & $\begin{array}{l}\text { Estudo } \\
\text { Experimental } \\
\text { The Importance } \\
\text { of Sleep: } \\
\text { Attentional } \\
\text { Problems in } \\
\text { School-Aged } \\
\text { Children With } \\
\text { Down Syndrome } \\
\text { and Williams } \\
\text { Syndrome }\end{array}$ & $\begin{array}{l}0 \text { estudo investigou } \\
\text { a associação entre } \\
\text { distúrbios } \\
\text { respiratórios do rono } \\
\text { e atenção em } \\
\text { crianças r com } \\
\text { síndrome de Down e } \\
\text { síndrome r de } \\
\text { Williams. }\end{array}$ & $\begin{array}{l}\text { O estudo associou a desatenção } \\
\text { aos distúrbios respiratórios do } \\
\text { sono em crianças com síndrome } \\
\text { de Down e síndrome de } \\
\text { William. Ressaltou a } \\
\text { importância de mais pesquisas } \\
\text { avaliando essa correlação. }\end{array}$ \\
\hline $\begin{array}{l}\text { Research in } \\
\text { Developmental } \\
\text { Disabilities. n. } \\
34, \quad \text { p.1572- } \\
\text { 1580, 2013. } \\
\text { ASHWORTH, A. } \\
\text { et al. }\end{array}$ & $\begin{array}{l}\text { Estudo } \\
\text { Experimental } \\
\text { Cross syndrome } \\
\text { comparison of } \\
\text { sleep problems } \\
\text { in children with } \\
\text { Down syndrome } \\
\text { and Williams } \\
\text { syndrome }\end{array}$ & $\begin{array}{l}\text { O estudo objetivou } \\
\text { expandir } \\
\text { conhecimento sobre } \\
\text { os problemas } \\
\text { respiratórios do sono } \\
\text { em crianças com } \\
\text { síndrome de Down e } \\
\text { síndrome } \\
\text { Williams. }\end{array}$ & $\begin{array}{l}\text { Os problemas do sono são comuns } \\
\text { em ambos os distúrbios do } \\
\text { desenvolvimento. Crianças com } \\
\text { síndrome de Down tiveram a maior } \\
\text { interrupção do sono, com } \\
\text { despertares frequentes e } \\
\text { inquietação. Além disso, há relatos } \\
\text { dos pais de bruxismo, ansiedade } \\
\text { do sono e resistência à hora de } \\
\text { dormir. As crianças com síndrome } \\
\text { de Williams apresentaram } \\
\text { problemas para iniciar o sono e os } \\
\text { pais também relataram enurese } \\
\text { noturna e dores musculares. }\end{array}$ \\
\hline
\end{tabular}




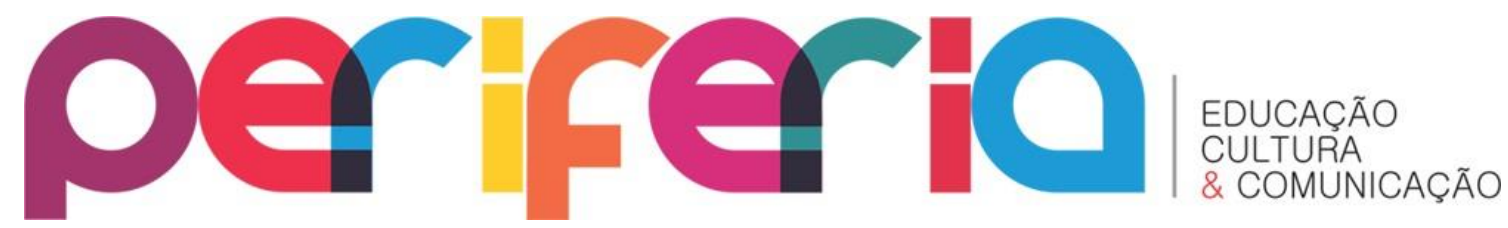

\begin{tabular}{|c|c|c|c|}
\hline $\begin{array}{l}\text { Exceptional } \\
\text { Children. v.79, } \\
\text { n.4, p. } 391, \\
2013 .\end{array}$ & $\begin{array}{l}\text { Estudo de } \\
\text { Revisão } \\
\text { Sistemática } \\
\text { Sleep and } \\
\text { cognitive } \\
\text { functioning in } \\
\text { children with } \\
\text { disabilities }\end{array}$ & 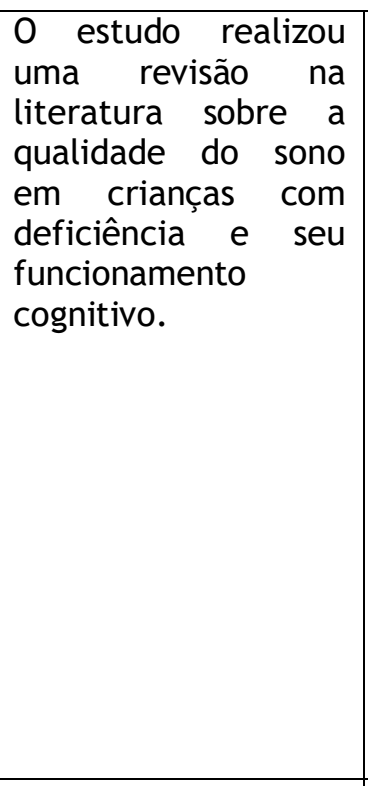 & $\begin{array}{l}\text { Os achados evidenciaram que } \\
\text { crianças com deficiência } \\
\text { (intelectual, transtorno de } \\
\text { déficit de atenção com } \\
\text { hiperatividade e transtorno do } \\
\text { espectro autista) têm padrões } \\
\text { atípicos de sono e alta } \\
\text { prevalência de distúrbios do } \\
\text { sono. Após uma descrição de } \\
\text { como o sono é avaliado, a } \\
\text { pesquisa relacionou o sono com } \\
\text { o funcionamento cognitivo nos } \\
\text { três grupos com deficiência. } 0 \\
\text { estudo sugeriu que outras } \\
\text { pesquisas avaliassem tas } \\
\text { implicações, para tanto, } \\
\text { recomendou tragem, } \\
\text { diagnóstico, prevenção na } \\
\text { educação. }\end{array}$ \\
\hline $\begin{array}{l}\text { Infants } \\
\text { Young } \\
\text { Children. v. 24, } \\
\text { n. 4, p. 295- } \\
\text { 308, 2011. } \\
\text { BONUCK, K. et } \\
\text { al. }\end{array}$ & $\begin{array}{l}\text { Estudo de } \\
\text { Revisão } \\
\text { Sistemática } \\
\text { Screening for } \\
\text { Sleep Problems } \\
\text { in Early } \\
\text { Intervention } \\
\text { and Early } \\
\text { Childhood } \\
\text { Special } \\
\text { Education: A } \\
\text { Systematic } \\
\text { Review of } \\
\text { Screening and } \\
\text { Assessment } \\
\text { Instruments }\end{array}$ & 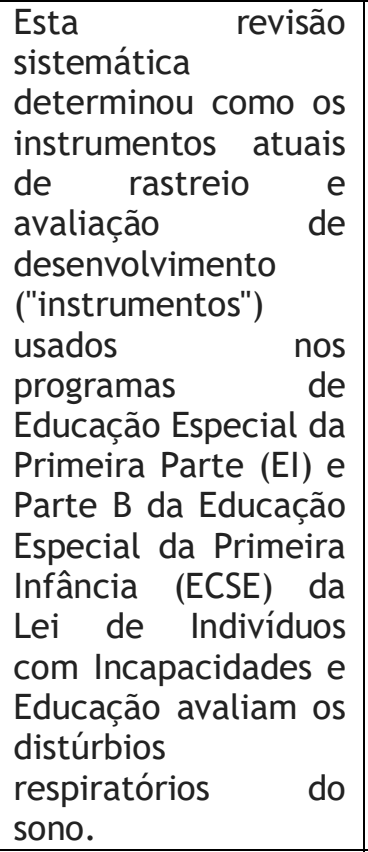 & $\begin{array}{l}\text { Foram analisados } 47 \text { únicos e } 67 \\
\text { no total (contemplando } \\
\text { múltiplas versões de idade e de } \\
\text { instrumentos únicos). Destes, } \\
\text { cerca da metade, nenhum item } \\
\text { relacionou o comportamento } \\
\text { aos distúrbios respiratórios do } \\
\text { sono. Ademais, daqueles que } \\
\text { fizeram, } 1,5 \% \text { dos itens, não } \\
\text { houve consistência para } \\
\text { relacionar os problemas } \\
\text { respiratórios do sono com as } \\
\text { comorbidades partilhadas no } \\
\text { problema do sono. Tal situação } \\
\text { é preocupante, haja vista a } \\
\text { necessidade de se intervir } \\
\text { precocemente junto aos } \\
\text { distúrbios do desenvolvimento } \\
\text { com programas de estimulação } \\
\text { essencial. }\end{array}$ \\
\hline $\begin{array}{l}\text { Journal of } \\
\text { Intellectual } \\
\text { Disability } \\
\text { Research. v. } \\
\text { 55, part. } 11, \mathrm{p} . \\
1086-1091, \\
2011 . \\
\text { BRESLIN. H. et }\end{array}$ & $\begin{array}{l}\text { Experimental } \\
\text { Parental Report } \\
\text { of Sleep } \\
\text { Problems in } \\
\text { Down Syndrome }\end{array}$ & 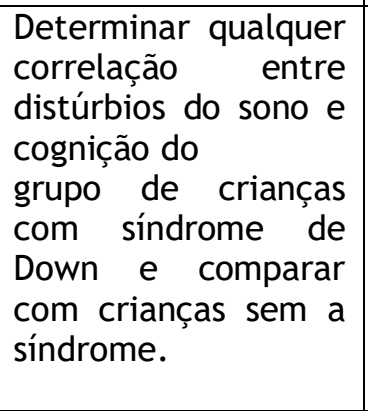 & $\begin{array}{l}\text { As crianças com síndrome de } \\
\text { Down estão em risco de } \\
\text { desenvolver } \\
\text { sintomas de }\end{array}$ \\
\hline $\begin{array}{ll}\text { Journal } & \text { of } \\
\text { Intellectual } & \\
\text { Disability } & \\
\text { Research. } & \text { v. } \\
53, \text { part. } 8 & \text { p. }\end{array}$ & $\begin{array}{l}\text { Estudo } \\
\text { Experimental } \\
\text { Sleep in } \\
\text { Individuals with }\end{array}$ & $\begin{array}{lr}\text { Verificar } & \text { a } \\
\text { ocorrência } & \text { de } \\
\text { problemas do sono } \\
\text { em pessoas com a } \\
\text { síndrome de Cri Du } \\
\end{array}$ & $\begin{array}{l}\text { Os achados revelaram que } \\
\text { indivíduos com Cri du Chat não } \\
\text { têm uma probabilidade maior } \\
\text { de apresentar problemas de } \\
\text { dormir comparados com outras }\end{array}$ \\
\hline
\end{tabular}




\section{OCO

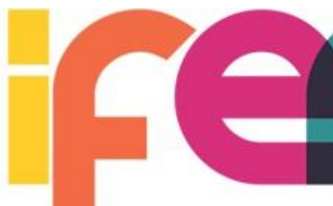

\begin{tabular}{|c|c|c|c|}
\hline $\begin{array}{l}\text { 704-715, } 2009 . \\
\text { MAAS, A. P. H. } \\
\text { M. et al. }\end{array}$ & $\begin{array}{lr}\text { Cri du } & \text { Chat } \\
\text { Syndrome: } & \text { A } \\
\text { Comparative } \\
\text { Study }\end{array}$ & $\begin{array}{l}\text { Chat e comparar } \\
\text { com indivíduos com } \\
\text { síndrome de Down e } \\
\text { deficiência } \\
\text { intelectual. }\end{array}$ & $\begin{array}{l}\text { pessoas de características } \\
\text { demográficas semelhantes. O } \\
\text { estudo levantou a hipótese de } \\
\text { problemas de vigília noturna em } \\
\text { pessoas com Cri du Chat e } \\
\text { ressalta a importância de mais } \\
\text { estudos avaliando esse dado. }\end{array}$ \\
\hline
\end{tabular}

Fonte: Elaborado a partir do levantamento de dados realizados pelas autoras.

Os resultados indicaram que a preocupação em inserir, na avaliação do desenvolvimento da pessoa com deficiência, a verificação da condição do sono desses é recente. A pesquisa de Bonuck et al. (2011), por exemplo, ressaltou que os distúrbios respiratórios do sono, na infância, quando não tratados, aumentam a probabilidade de risco de desenvolvimento, sobretudo, no comportamento, cognição e crescimento.

Desta forma, o estudo que Bonuck et al. (2011) realizaram, nos Estados Unidos, foi uma revisão sistemática dos instrumentos de rastreio e avaliação de desenvolvimento nos programas de Educação Especial da primeira fase (El) e da parte B da Educação Especial da Primeira Infância (ECSE) para verificar se esses materiais contemplavam em seu protocolo algum item referente aos problemas do sono. Para os lactentes e crianças pequenas, os referentes e as insônias comportamentais da infância (BIC), enquanto que para as crianças maiores, os distúrbios respiratórios desordenados (SDB), ou seja, ronco e apneia obstrutiva do sono.

A equipe da pesquisa supracitada analisou 47 instrumentos exclusivos e 67 de versões de vários anos de instrumentos únicos. A análise revelou que $47 \%$ dos instrumentos exclusivos e $38 \%$ do total não continham itens relacionados ao sono. Nenhum (0\%) protocolo incluiu qualquer item sobre SDB. Os itens mais citados foram: "resistência ao deitar"; "vigília noturna" e "preocupações dos pais com relação ao sono da criança". Contudo, menos de $20 \%$ os instrumentos abordaram até mesmo esses itens. Dentre os 10 instrumentos mais utilizados nos programas El e ECSE, somente $40 \%$ 


\section{periferio}

apresentava itens do tipo BIC e nenhum fazia menção sobre os sinais e sintomas SDB.

Outra pesquisa que teve a preocupação em realizar um levantamento sistemático sobre o sono e o funcionamento cognitivo em crianças com deficiência foi a de Buckhalt (2013). 0 estudo realizou um levantamento entre as crianças com deficiência em geral, incluindo a deficiência intelectual e os Transtorno de Déficit de Atenção com Hiperatividade e Transtorno do Espectro Autista (TEA).

No levantamento de Buckhalt (2013), o autor citou a pesquisa de Kopasz et al. (2010) que revisou 15 estudos que relacionaram sono, medidas de atenção, aprendizagem e memória. Destacou que a maioria dos estudos reforçam a hipótese de que o sono é um aliado importante na consolidação da memória em crianças e adolescentes. Ademais, ressaltou que o desempenho em tarefas abstratas e complexas diminui consideravelmente em escolares com privação do sono.

Em relação aos distúrbios do neurodesenvolvimento, o mapeamento realizado por Buckhalt (2013) verificou problemas de sono associados ao Transtorno do Espectro Autista (TEA), à síndrome de Down (SD) e à síndrome de Rett. Em relação ao sono das pessoas com transtornos invasivos do desenvolvimento, Buckhalt (2013) relatou que a literatura aponta associações importantes entre esses, sobretudo, os comportamentos de internalização e externalização, sintomas afetivos, comportamento repetitivo, comportamento adaptativo, linguagem receptiva e inteligência.

Buckhalt (2013) destacou que a prevalência de apneia obstrutiva do sono para os sujeitos com síndrome de Down está entre $30 \%$ e $80 \%$. 0 pesquisador sinalizou que tal incidência pode estar relacionada ao desenvolvimento neurológico que apresenta anormalidades craniofaciais, hipotonia e obesidade. Para as pessoas com síndrome de Rett, a apneia do sono não é tão comumente observada quanto na síndrome de Down. No entanto, os cuidadores registraram frequentes problemas associados, como 


\section{periferio}

despertares noturno, queda da cama e continuidade do sono e sonolência diurna.

Breslin et al. (2011) investigaram a relação entre sono e cognição de 35 pessoas com síndrome de Down, com idade média de 12,65 anos. Para avaliar a qualidade do sono desses participantes, os responsáveis responderam a um questionário padronizado. 0 Questionário de Hábitos de Dormir das Crianças (CSHQ) é um instrumento de triagem que contempla 33 itens que avalia os principais domínios do sono, tanto em nível médico quanto comportamental.

Os resultados do estudo citado revelaram que $20 \%$ da amostra necessitava de um acompanhante para dormir, 28,5\% necessitava de pelo menos duas vezes por semana de um acompanhante para dormir e $34 \%$ apresentava dificuldade em adormecer. Em repouso, 43\% foi descrito "às vezes" agitados e $37 \%$ com frequência agitados durante o sono. Algumas parassonias do sono foram registradas, como bruxismo (34\%) e fala durante o sono (29\%). Além disso, em $60 \%$ da amostra pelo menos um sintoma de distúrbios respiratórios do sono foi identificado, incluindo o ronco alto, ronco ofegante e cessação de respiração durante o sono. Em relação às consequências diurnas da privação da qualidade do sono, 56,3\% apresentavam sonolência durante o dia e $57 \%$ adormeciam rotineiramente enquanto assistiam televisão e 60\% dormiam quando andavam de carro.

Destarte, o estudo de Breslin et al. (2011) constatou que os problemas do sono eram bastante comuns nessa população (SD), podendo apresentar problemas adicionais que não estão relacionados à respiração desordenada do sono.

$\mathrm{Na}$ busca por detectar os problemas adicionais dos distúrbios respiratórios do sono, a pesquisa de Rahmawati et al. (2015) investigou a relação entre posturas do sono e parâmetros de distúrbios respiratórios do sono em pessoas com síndrome de Down no Japão. Na pesquisa, os autores ainda ressaltaram que essas crianças apresentam resistência à hora de dormir, sono agitado, enurese durante o sono, despertares noturnos e sonolência diurna. 


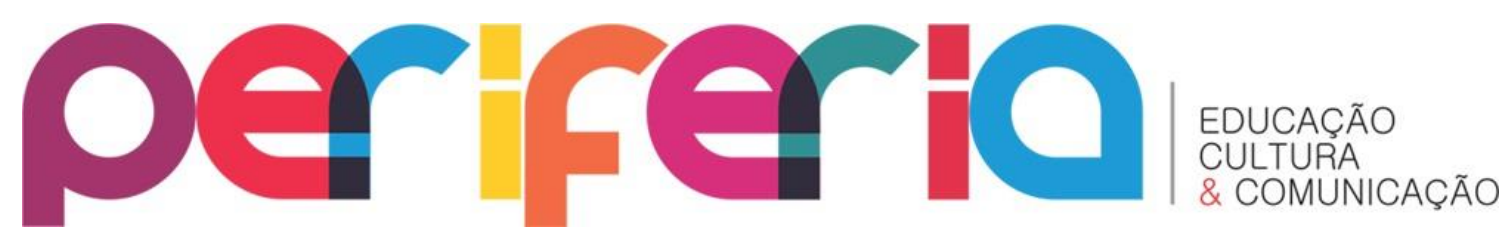

Rahmawati et al. (2015) destacaram que, de acordo com a Academia Americana de Medicina do Sono e a Academia Americana de Pediatria, o padrão-ouro para avaliar os problemas do sono é o exame de polissonografia noturna. Contudo, mencionaram que outra técnica que possibilita a avaliação e registro da atividade motora por meio dos movimentos é a actigrafia. Esta, por meio de um dispositivo que é acoplado no pulso (semelhante a um relógio), monitora a oximetria de pulso, registrando o movimento desde o momento de ir para a cama até levantar-se. Os estudiosos ressaltaram que esse procedimento é minimamente invasivo cuja sensibilidade foi de $86,6 \%$ e especificidade de $98,9 \%$.

Desta forma, participaram da pesquisa de Rahmawati et al. (2015) 36 pessoas com SD e seus respectivos responsáveis que responderam a um questionário e concordaram da gravação de oximetria de pulso.

Os resultados do estudo citado reforçaram os achados de pesquisas anteriores sobre os sinais e sintomas dos distúrbios respiratórios do sono, noturno e diurno. A investigação entre a postura do sono existente, a dessaturação noturna de oxigênio em pessoas com síndrome de Down e a relação entre as características dos distúrbios do sono que afetam essas posturas indicaram que as posturas de sono em prono (abdômen para baixo) foram incomuns em SD.

Os pesquisadores ressaltaram que a postura prono é uma compensação contra a respiração dos distúrbios respiratórios do sono, principalmente, em crianças pequenas, pois essas posturas podem estar relacionadas ao alívio dos distúrbios respiratórios do sono, bem como à imaturidade do sistema respiratório de muitas pessoas com SD. Além disso, destacaram que a posição de preferência adotada por esses é de dobra, ou seja, sentada na cama de pernas cruzadas com a cabeça projetada para frente e abaixada. Os autores supracitados inferiram que a razão para esta postura pode estar relacionada ao mecanismo de proteção contra a dessaturação noturna de oxigênio.

Outro estudo realizado com SD foi o do grupo de pesquisadores ingleses (ASHWORTH et al., 2013). Esta equipe comparou, pela primeira vez, o sono de 


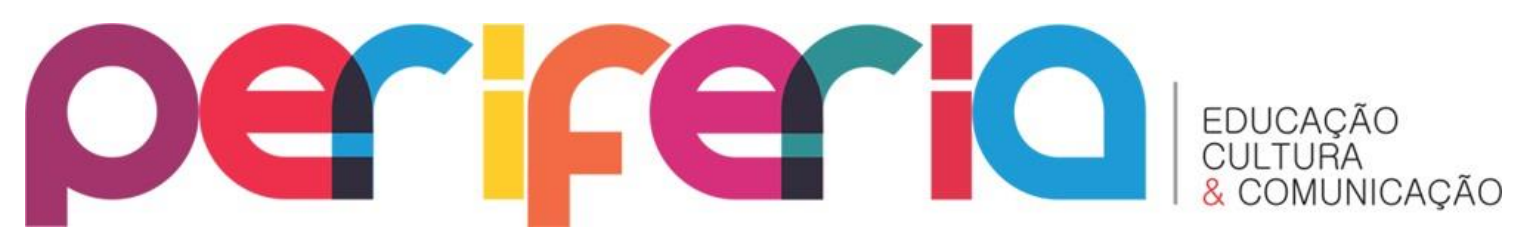

crianças com $S D(N=22)$, síndrome de Williams - SW $(N=24)$ e desenvolvimento típico (DT) ( $N=52)$ por meio de um dispositivo (actigrafia) acoplado no pulso desses participantes e questionário respondido por seus respectivos cuidadores. Participaram da pesquisa somente as crianças, dos três grupos que não apresentavam problemas médicos ou psiquiátricos, os quais pudessem interferir no sono, bem como àquelas que faziam uso de medicamentos controlados, como para asma e epilepsia. Também foram excluídas do grupo crianças cujo inglês não fosse sua primeira língua.

De acordo com os pesquisadores (ASHWORTH, et al., 2013), a comparação entre síndromes e um grupo controle de crianças com desenvolvimento típico determinaria se existem padrões específicos para cada transtorno. Um achado interessante nesse estudo foi que não houve nenhuma relação significativa entre o relato dos cuidadores e a actigrafia. As correlações foram negativas, sugerindo que o nível de conhecimento dos responsáveis era limitado sobre o sono das crianças (ASHWORTH, et al., 2013. Ressalta-se que tal dado é muito importante, pois muitas pesquisas que avaliam o sono em crianças se embasam apenas em um preenchimento de questionário aplicado junto aos pais (ARAUJO, 2012).

Desta forma, a pesquisa de Ashworth et al. (2013) ratificou que problemas de sono são comuns na SD e SW, destacando a necessidade de avaliação objetiva em grupos com desenvolvimento atípicos. Os autores supracitados ressaltaram que esta foi a primeira vez em que o sono dessas síndromes foi comparado. No entanto, salientaram que no grupo com SD os problemas do sono foram mais observados, bem como sua fragmentação. Tal situação repercutia durante o dia, a qual se manifestava com sonolência excessiva diurna.

A comparação com os três grupos revelou que as crianças sindrômicas são muito mais propensas a se mudarem para a cama de noite do que as com desenvolvimento típico. Contudo, a enurese noturna e a queixa de dor no corpo foram problemas mais observados no grupo com SW. A inquietação, o 


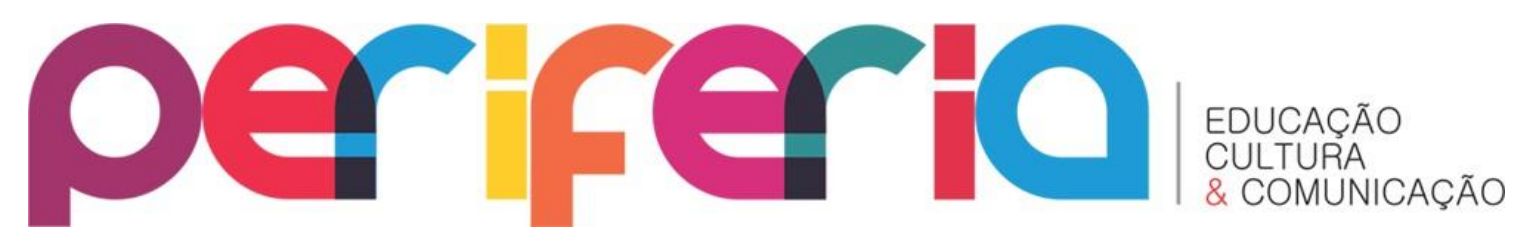

bruxismo, o ronco alto e acordar cedo foram sinais mais observados nas crianças com SD.

Dando continuidade ao esclarecimento dos distúrbios respiratórios do sono na população com SD e SW, Ashworth et al. (2014) investigaram a associação entre distúrbios respiratórios do sono e atenção nesses dois grupos. Participaram do estudo 22 crianças com SD, 22 escolares com SW e 41 alunos com desenvolvimento típico. Esses estudantes foram recrutados através de escolas primárias na cidade de Londres, Inglaterra. Os educandos com SD foram selecionados por meio de escolas para crianças com necessidades especiais e os com SW foram indicados pela Willians Syndrome Foundation, Reino Unido.

Os responsáveis de todas as crianças participantes da pesquisa supracitada informaram sobre a saúde geral de seus filhos e assinaram o Termo de Consentimento Livre e Esclarecido. Foram excluídos do estudo os sujeitos que apresentavam transtornos comórbidos, psiquiátricos e aqueles que faziam uso de medicação hipnótica.

Para avaliar os padrões do sono dos três grupos (SD, SW, DT) foi utilizada a actigrafia. Para verificar a inteligência fluida foi utilizado o Teste Raven's Colored Progressive Matrices, cujo instrumento é frequentemente utilizado para pontuar o raciocínio não verbal das crianças.

A atenção (seletiva e sustentada) e a impulsividade dos grupos foram aferidas por meio da Tarefa de Atenção de Desenvolvimento Contínuo. A tarefa foi exibida na tela do computador e exigia do participante a precisão do tempo de reação frente ao solicitado, por exemplo, responder aos estímulos alvos e ignorar os estímulos distratores. Os estímulos eram imagens coloridas de animais de jardim zoológico nos quais os alvos eram dois macacos diferentes entre outros oito animais diferentes (leão, tigre, tamanduá, leopardo, girafa, elefante, hipopótamo e polvo).

A relação entre sono e atenção em educandos com SD e SW em comparação com DT revelaram problemas de sono nos dois grupos de crianças com deficiência. 0 desempenho na tarefa de atenção ratificou os achados de 


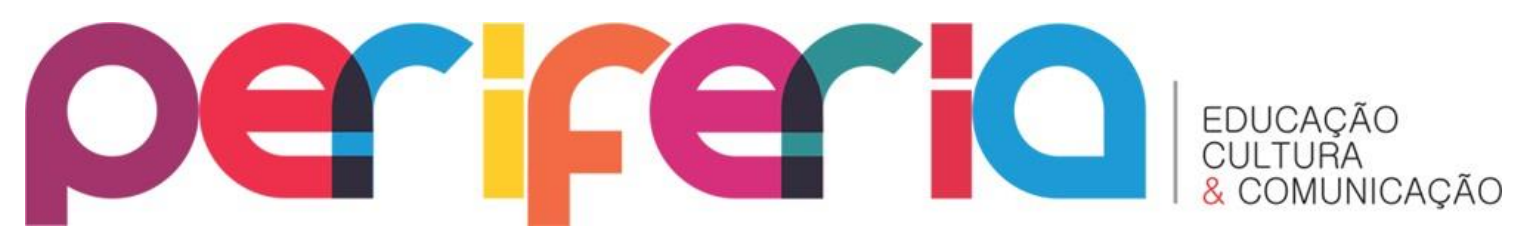

pesquisas anteriores sobre problemas atencionais em crianças com SD e SW. Contudo, o grupo com SD apresentou desempenho significativamente menor do que os outros dois grupos na habilidade de responder ao alvo. Esta pesquisa foi a primeira a investigar os aspectos do sono e de atenção em escolares com SD e SW e salientou a necessidade de se investir em estudos futuros para melhor contribuições da atenção, do sono e sua influência nesses dois grupos (ASHWORTH et al., 2014).

Como constatado, os problemas do sono são comuns em pessoas com deficiência intelectual, sobretudo, na síndrome de Down. Um grupo de pesquisadores holandeses (MAAS et al., 2009) foram os primeiros estudiosos a investigarem a ocorrência de problemas do sono em pessoas com a síndrome de Cri Du Chat $(\mathrm{N}=30)$ em comparação aos indivíduos com síndrome de Down $(N=30)$ e deficiência intelectual não especificada $(N=30)$. 0 sono dos três grupos foi avaliado por meio de um questionário padronizado.

A comparação dos três grupos, por meio do questionário padronizado do sono, revelou que 30\% do grupo com síndrome de Cri Du Chat (CDC) apresentou problemas de sono graves ou leves, assim como problemas com a vigília noturna frequente. Os pesquisadores concluíram que o grupo com CDC não tem maior incidência de problemas de sono, se comparados com outros indivíduos que compartilham semelhantes características demográficas.

A equipe de Maas et al. (2009) ressaltou que foram observados no grupo de CDC uma diversidade de problemas médicos decorrentes da própria síndrome que poderiam contribuir para a ocorrência de noites mal dormidas, como tosse/resfriados, escoliose e problemas de constipação.

Neste sentido, os pesquisadores (MAAS et al., 2009) pontuaram que, apesar de o estudo ter contribuído para o esclarecimento, há diferenças significativas de problemas de sono nos três grupos participantes, alguns limites metodológicos foram observados. A primeira lacuna refere-se ao método de pesquisa. Apenas a aplicação do questionário junto aos responsáveis torna-se o estudo muito subjetivo. Dados adicionais foram importantes para maiores esclarecimentos dos problemas do sono, como um 


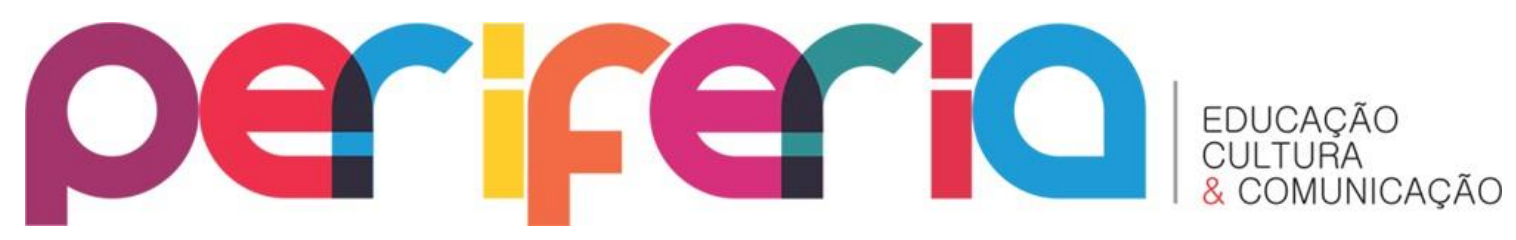

método objetivo poderia ser a utilização da actigrafia, pois teria ampliado o conhecimento sobre os padrões do sono nos três grupos. Outra deficiência do estudo diz respeito à pequena dimensão da amostra, tornando difícil a exploração da associação entre indivíduos, variáveis, problemas de sono, comportamentos relacionados ao sono e aos tipos de distúrbios do sono entre grupos.

Contudo, apesar das deficiências observadas na pesquisa de Maas et al. (2009), essa forneceu pistas para que novos estudos venham ampliar o conhecimento dos distúrbios do sono na síndrome de CDC com vistas a melhorar a qualidade do sono do grupo.

Do levantamento realizado para este estudo, evidenciou-se que as pesquisas referentes aos distúrbios do sono em escolares com desenvolvimento típico, apesar do aumento de estudos consideráveis dos últimos anos, ainda necessita de muitas investigações para esclarecer melhor as comorbidades dos distúrbios do sono no desenvolvimento e aprendizagem das crianças e adolescentes (DI FRANCESCO, 2004; LUMENG; CHERVIN, 2008; BECKER; FRANCO; ANJOS, 2013).

No entanto, observou-se que há caracterísicas comuns aos dois grupos com se sem deficiência, tais como ronco, agitação noturna, irritação, respiração ruidosa, pausas respiratórias, déficit de atenção, instabilidade de humor e dificuldade de socialização, cooperação (MARTINEZ, 1999; GOTTLIEB; CHASE; VEZIN, 2004; VALERA; DEMARCO; ANSELMO-LIMA, 2004; BRESLIN et al., 2011; ASHWORTH, et al., 2013; BUCKHALT, 2013).

Huang et al. (2007) verificaram que os distúrbios respiratórios do sono (DRS) prejudicam a atenção e o comportamento das crianças com desenvolvimento típico, o que pode ocasionar a confusão de diagnóstico, como o Transtorno por Déficit de Atenção/Hiperatividade (TDAH). Ashworth e sua equipe (2014) também verificaram problemas atencionais em escolares com síndrome de Down e Síndrome de Williams com DRS, ratificando a influência dos problemas do sono nos aspectos atencionais. 


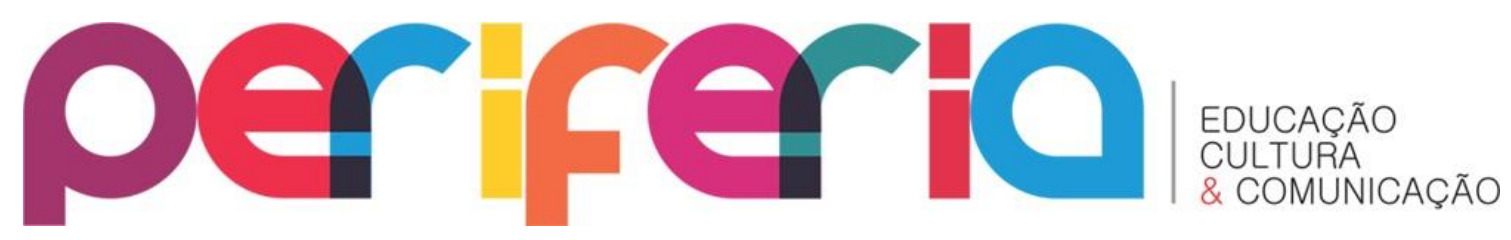

Observou-se que o exame indicado para avaliar os DRS é a polissonografia (MACEDO; COLOMBINI; PINTO, 2010). Porém, devido aos elevados custos do exame, muitas pesquisas têm avaliado a qualidade do sono por meio de questionários padronizados do sono. Tal situação tem sido recorrente no diagnóstico dos escolares com e sem deficiência (MAAS et al., 2009; BRESLIN, 2011). Outro instrumento que os pesquisadores dos distúrbios do sono têm utilizado para avaliação das crianças e adolescentes com deficiência é a actigrafia. Apesar de não ser o padrão ouro para esse tipo de diagnóstico, a mesma contribui para ampliar o conhecimento sobre a qualidade do sono do avaliado (RAHMAWATI et al., 2015).

Assim como Bonuck et al. (2011), em sua pesquisa, observaram a carência de avaliação do sono quando do diagnóstico psicoeducacional de escolares com desenvolvimento típico americano, destacando-se a necessidade de inserir nos programas de Educação Especial brasileiro a avaliação do sono de crianças com deficiência.

\section{CONCLUSÃO}

A revisão sistemática revelou que as pesquisas sobre a influência dos distúrbios do sono junto às crianças e aos adolescentes com deficiência intelectual é recente e, ainda, limitada. Apesar dos esforços dos pesquisadores em trazerem a temática, distúrbios do sono, para a área da Educação Especial há uma lacuna muito extensa a ser preenchida, sobretudo, em relação às pesquisas empíricas com tal população e seus pares, por exemplo, estudos controle entre sujeitos com síndrome de Down com e sem distúrbios do sono e a avaliação da memória, atenção e desempenho escolar.

Desta forma, esse levantamento ressaltou a necessidade das pesquisas brasileiras, sobretudo em Educação Especial, de se preocuparem em inserir os distúrbios respiratórios do sono nos estudos junto a essa população escolar.

Acredita-se que esse levantamento estendeu a literatura sobre os distúrbios respiratórios do sono, no sentido de alertar os estudiosos nacionais 


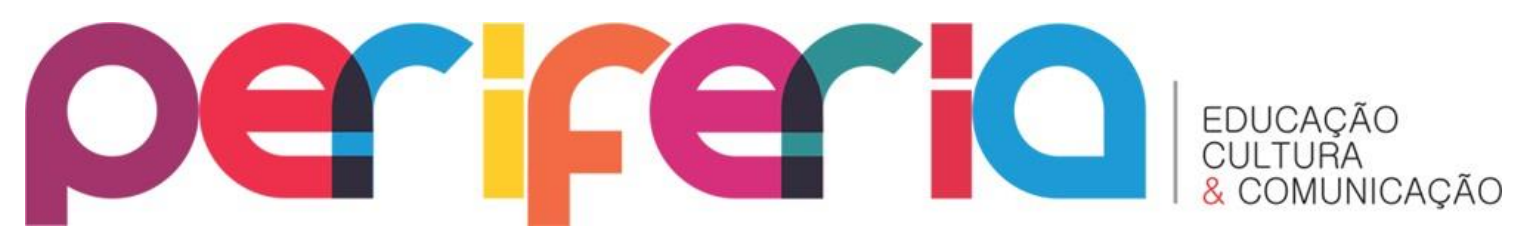

a inserirem no escopo de suas pesquisas essa temática. Sendo assim, poder-seá ampliar o debate e ajudar a melhorar a qualidade de vida e de aprendizagem de educandos Público Alvo da Educação Especial.

\section{REFERÊNCIAS}

ANSELMO-LIMA, W. L.; TAMASHIRO, E.; VALERA, F. C. P. A criança respiradora oral (epidemiologia, quadro clínico e etiologia). In: SOLÉ, D.; PRADO, E.; WECKX, L. L. M (Orgs.). Obstrução nasal: o direito de respirar pelo nariz. São Paulo: Editora Atheneu, 2013.

ARAÚJO, P. D. P. Validação do questionário do sono infantil de Reimão e Lefévre (QRL). 2012. 123f. Tese (Doutor em Ciências) - Faculdade de Medicina da Universidade de São Paulo. São Paulo, 2012.

ASHWORTH, A. et al. The Importance of Sleep: Attentional Problems in School-Aged Children With Down Syndrome and Williams Syndrome. Behavioral Sleep Medicine, n. 12, p.1-17, 2014.

ASHWORT, A. et al. Cross syndrome comparison of sleep problems in children with Down syndrome and Williams syndrome. Research in Developmental Disabilities, n. 34, p.1572-1580, 2013.

BECKER, H. M. G.; FRANCO, L. P.; ANJOS, C. P. G. dos. Aprendizado e o respirador oral. In: SOLÉ, D.; PRADO, E.; WECKX, L. L. M (Orgs.). Obstrução nasal: o direito de respirar pelo nariz. São Paulo: Editora Atheneu, 2013.

BONUCK, K. et al. Screening for Sleep Problems in Early Intervention and Early Childhood Special Education A Systematic Review of Screening and Assessment Instruments. Infants \& Young Children, v. 24, n. 4, p. 295-308, 2011.

BRANDAU, R.; MONTEIRO, R.; BRAILE, D. M. Importância do uso correto dos descritores nos artigos científicos. Revista Brasileira de Cirurgia Cardiovascular, v. 20, n.1, São José do Rio Preto Jan./Mar. 2005.

BRESLIN, J. H. et al. Parental report of sleep problems in Down syndrome. Journal of Intellectual Disability Research, v. 55, part. 11, p. 1086-1091, 2011.

BUCKHALT, J. et al. Sleep and cognitive functioning in children with disabilities. Exceptional Children, v.79, n.4, p. 391, 2013. 


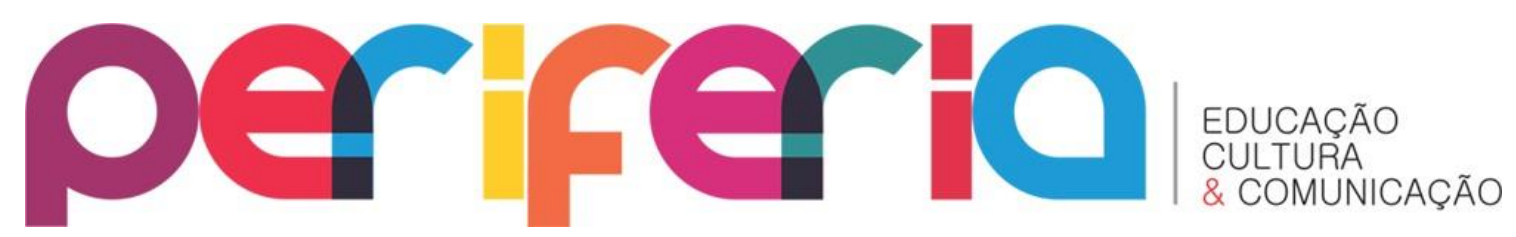

COSTA, A. B.; ZOLTOWSKI, A. P. C. Como escrever um artigo de revisão sistemática. In: KOLLER, S.; COUTO, M. C. P. P.; HOHENDORFF, J. V. (Orgs.). Manual de produção científica. Porto, Alegre: Penso, 2014.

DI FRANCESCO, R. C. et al. Respiração oral na criança: repercussões diferentes de acordo com o diagnóstico. Revista Brasileira de Otorrinolaringologia, v. 70, n. 5, 2004.

GOTTLIEB, D. J.; CHASE, C. VEZINA, R. M. Sleep-disordered breathing symptoms are associated with poorer cognitive function in 5-year-old children. Journal Pediatrics, Illinois, v.145, p. 458-64, 2004.

GOZAL, D. Sleep-disordered breathing and school performance in children. Journal Pediatrics, Illinois, v. 102, n.3, p. 616-620, 1998.

.; POPE, D.W. Snoring during early childhood and academic performance at ages thirteen to fourteen years. Journal Pediatrics, Illiniois, v.107, n. 6, 1394-1399, 2001.

GUILLEMINAULT, C; ELDRIDGE, F. L.; SIMMONS F. B.; DEMENT, W. C. Sleep apnea in eight children. Journal Pediatrics, Illinois, v. 58, p. 23-30, 1976.

HOHENDORFF, J. Como escrever um artigo de revisão de literatura. In: . (Org.). Publicar em psicologia: um enfoque para a revista científica. São Paulo: Associação Brasileira de Editores Científicos de Psicologia/ instituto de Psicologia da Universidade de São Paulo, 2009, p. 3954.

HUANG, H. S.; GUILLEMINAULT, C.; LI, H. Y.; YANG, C. M.; WU, YY.; CHEN, N. $\mathrm{H}$. Attention-deficit/hyperactivity disorder with obstructive sleep apnoea: a treatment outcome study. Journal Sleep Medicine, Darien, v. 8, p. 18-30, 2007.

LUMENG, J. C.; CHERVIN, R. D. Epidemiology of pediatric obstructive sleep apnoea. Proceedings of the American Thoracic Society, Michigan, v. 5, n. 2, p. 242-52, 2008.

MACEDO, M. M. C.; COLOMBINI; N. E. P.; PINTO, J. A. Síndrome da apneia obstrutiva do sono (SAOS) e outros disturbios respiratórios: enfoque craniomaxilofacial. In: COLOMBINI, N.; MACEDO, M. Do respirador bucal à apneia obstrutiva do sono: enfoque multidisciplinar. São Paulo: Ícone, 2010.

MARTINEZ, D. Prática da medicina do sono. São Paulo: BYK, 1999. 


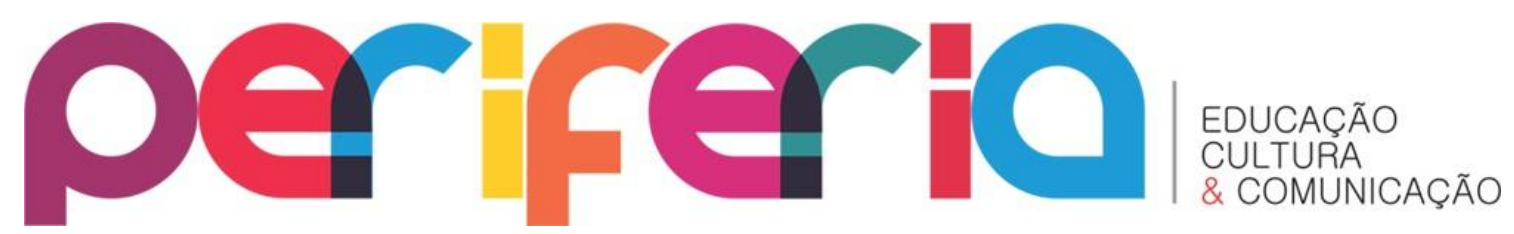

MASS, A. P. H. M. et al. Sleep in individuals with Cri du Chat syndrome: a comparative study. Journal of Intellectual Disability Research. v. 53, part. 8 p. 704-715, 2009

OWENS, J. A. A clinicalguide to pediatric sleep: diagnosis and management of sleep problems. Philadelphia, PA: Lippincott, Williams \& Wilkins, 2010.

RAHMAWATI, A. Relationship between sleep postures and sleep-disordered breathing parameters in people with Down syndrome in Japan. Sleep and Biological Rhythms, n.13, p. 323-331, 2015.

ROSINHA, M. U. Fisiología do sono: atualizações. In: REIMÃO, R. Temas de medicina do sono. São Paulo: Lemos Editorial, 2000.

SILVEIRA, K. A.; ENUMO, S. R. F.; ROSA, E. M. Concepções de professores sobre inclusão escolar e interações em ambiente inclusivo: uma revisão da literatura. Revista Brasileira de Educação Especial, Marília, v.18, n.4, p.695708, 2012.

SPAHIS, J. Sleepless nights: obstructive sleep apnea in the pediatric patient. Journal Pediatric Nursing, Philadelphia, v. 20, n. 5, p. 469-472, 1994.

VALERA, F. C. P; DEMARCO, R. C.; W. T. ANSELMO-LIMA. Síndrome da Apnéia e da Hipopnéia Obstrutivas do Sono (SAHOS) em crianças. Revista Brasileira de Otorrinolaringologia, São Paulo, v.70, n.2, p. 232-237, 2004. 